\title{
Classification of Normal and Crackles Respiratory Sounds into Healthy and Lung Cancer Groups
}

\author{
N. Abdul Malik, W. Idris, T. S. Gunawan, R. F. Olanrewaju, S. Noorjannah Ibrahim \\ Departement of Electrical and Computer Engineering, International Islamic University Malaysia, Malaysia
}

\begin{tabular}{l}
\hline \hline Article Info \\
\hline Article history: \\
Received Feb 19, 2018 \\
Revised Apr 2, 2018 \\
Accepted Apr 10, 2018 \\
\hline
\end{tabular}

Keyword:

ANN

Crackles

DWT

Lung cancer

Respiratory sound

\begin{abstract}
Lung cancer is the most common cancer worldwide and the third most common cancer in Malaysia. Due to its high prevalence worldwide and in Malaysia, it is an utmost importance to have the disease detected at an early stage which would result in a higher chance of cure and possibly better survival. The current methods used for lung cancer screening might not be simple, inexpensive and safe and not readily accessible in outpatient clinics. In this paper, we present the classification of normal and crackles sounds acquired from 20 healthy and 23 lung cancer patients, respectively using Artificial Neural Network. Firstly, the sounds signals were decomposed into seven different frequency bands using Discrete Wavelet Transform (DWT) based on two different mother wavelets namely Daubechies 7 (db7) and Haar. Secondly, mean, standard deviation and maximum PSD of the detail coefficients for five frequency bands (D3, D4, D5, D6, and D7) were calculated as features. Fifteen features were used as input to the ANN classifier. The results of classification show that $\mathrm{db} 7$ based performed better than Haar with perfect $100 \%$ sensitivity, specificity and accuracy for testing and validation stages when using 15 nodes at the hidden layer. While for Haar, only testing stage shows the perfect $100 \%$ for sensitivity, specificity, and accuracy when using 10 nodes at the hidden layer.
\end{abstract}

Copyright $@ 2018$ Institute of Advanced Engineering and Science. All rights reserved.

\section{Corresponding Author:}

N. Abdul Malik,

Department of Electrical and Computer Engineering,

International Islamic University Malaysia,

Jalan Gombak, 53100 Kuala Lumpur, Malaysia.

Email: norehaa@iium.edu.my

\section{INTRODUCTION}

According to International Agency for Research on Cancer (IARC) [1], lung cancer is the most common cancer worldwide with more than 1.8 million new cases and 1.6 million deaths estimated in 2012. While in Malaysia, it is the third most common cancer after colorectal and breast cancers. There were 10,608 cases reported by Malaysian National Cancer Registry between 2007 and 2011 [2]. Majority of the patients present later stage of the disease ie. stage 3 or 4 diseases and therefore curative treatment is seldom an option and prognosis is poor. Due to its high prevalence in Malaysia, it is an utmost importance to have the disease detected at an early stage which would result in a higher chance of cure and possibly better survival. Sputum cytology and chest X-ray (CXR) has been used for screening of lung cancer and recently low radiation-dose helical CT (spiral CT) has been shown to be superior to conventional CXR [3]. Autofluorescence bronchoscopy is also one of the potential screening tools [4]. However, all the mentioned tests might not be simple, inexpensive and safe and not readily accessible in outpatient clinics.

Auscultation is a non-invasive, safe and inexpensive technique used to listen to lung and heart sounds. It is performed as clinical examination and it can provide useful information regarding lung condition. Computerized auscultation has overcome limitations of the traditional technique that uses an analog stethoscope. Classifications of respiratory sound through computerized auscultation have shown 
promising results for the diagnosis of various lung diseases [5]. Based on the review paper, lung sound analyses using computerized auscultation gave good results of sensitivity and specificity. Most of the analyses pre-processed the sound signal to reduce noises and extract useful features and use machine learning for classification. There were different filtering techniques used to reduce heart sounds from lung sounds such as wavelet transform [6], adaptive filtering [7], [8] and bandpass filtering [8]. For further analysis, fast Fourier transform (FFT) [9], short time Fourier transform (STFT) [10] or discrete wavelet transform (DWT) [11] has been applied to transform the signals into different domain such as frequency or time-frequency domain. Spectral representation of the signal makes it more useful to extract features required by learning algorithms.

Respiratory sounds can be classified into normal and adventitious sounds. The adventitious sound can be further classified as discontinuous and continuous based on their characteristics [12]. For instance, crackles either fine or coarse are classified as discontinuous adventitious sounds while wheezes and rhonchus belong to continuous adventitious sounds [13]. Several studies have analysed respiratory sounds in asthma, pneumonia, chronic obstructive pulmonary disease (COPD) and idiopathic pulmonary fibrosis patients to characterize and classify as normal, wheeze, rhonchi, coarse crackles and fine crackles [11], [13]. Different types of classification methods have been employed to classify crackles for examples using Tsallis Entropy and Multilayer Perceptron [14], K-nearest Neighbor [15] and Support Vector Machine (SVM) [16].

Although many researchers have classified crackles sound, none has taken samples from lung cancer patients from the best knowledge of the authors. Only [13] have used samples from lung cancer but the samples were taken randomly from various pulmonary diseases for the analysis. In this study, the crackles sounds are extracted from lung cancer patients only.

\section{METHODOLOGY}

This section presents the methodology used in this study to classify between normal and crackles sounds in healthy and lung cancer patients. The proposed algorithm is shown in Figure 1.

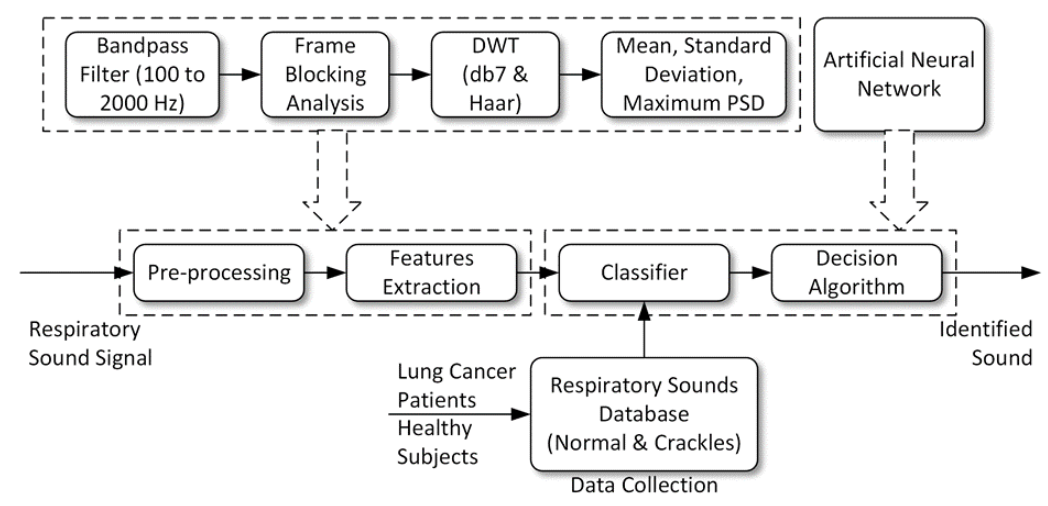

Figure 1. Proposed Algorithm using DWT to Identify Normal and Crackles in Healthy and Lung Cancer Patients

\subsection{Data collection and pre-processing of respiratory sounds}

Data collection for this study was approved by medical ethics committee of University Malaya Medical Centre (UMMC) with reference number (MREC ID NO: 201698-4242). There were 20 normal subjects and 23 lung cancer patients participated in the data collection which took place at Clinical Oncology Unit, University Malaya Medical Centre. The participated patients have no co-existing respiratory related diseases. All the healthy subjects recruited are the non-smoker. All the subjects were given inform consent form and briefed on the study protocol. The respiratory sound of normal subjects and lung cancer patients was acquired using digital stethoscope by Thinklabs and saved as .au in a computer laptop using Thinklabs Phonocardiography by Audacity. The stethoscope was connected to the computer laptop via a sound card (Xonar U3) and the computer was disconnected from the main power supply during the recording. The sampling rate used was $11025 \mathrm{~Hz}$. All the subjects were asked to breathe normally and the respiratory sound was recorded for about 20 seconds for each auscultation point. In total, there were twenty-two auscultation points, eleven points each at anterior and posterior of the chest wall including trachea as shown in Figure 2(a) and Figure 2(b). 


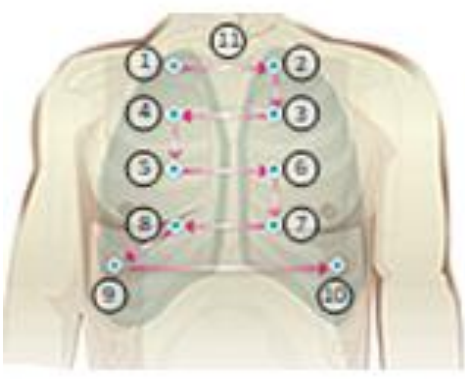

(a)

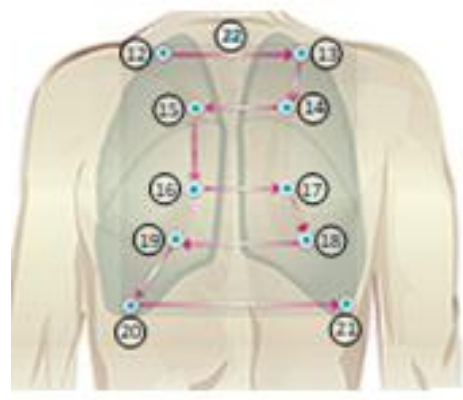

(b)

Figure 2. Auscultation points (a) eleven points anterior (b) eleven points posterior

Next, a bandpass filter with cut-off frequencies of 100 and $2000 \mathrm{~Hz}$ was applied to the raw respiratory sound signal to enhance the lung sound using Thinklabs Phonocardiography software. Filtering process is needed to reduce noises coming from the heart, muscle or ambient which are not related to the lung sound. Crackles sounds present in the lung cancer patient's respiratory sound were identified manually. The respiratory sound cycle consists of crackles is extracted and exported as .wav to be read later by MATLAB for signal decomposition and feature extraction processes. There were 60 samples consist of crackles sound and 60 samples of normal sounds. Figure 3(a) and Figure 3(c) show the respiratory sounds cycle of lung cancer patient and healthy subject before filtering while Figure 3(b) and Figure 3(d) after filtering, respectively.
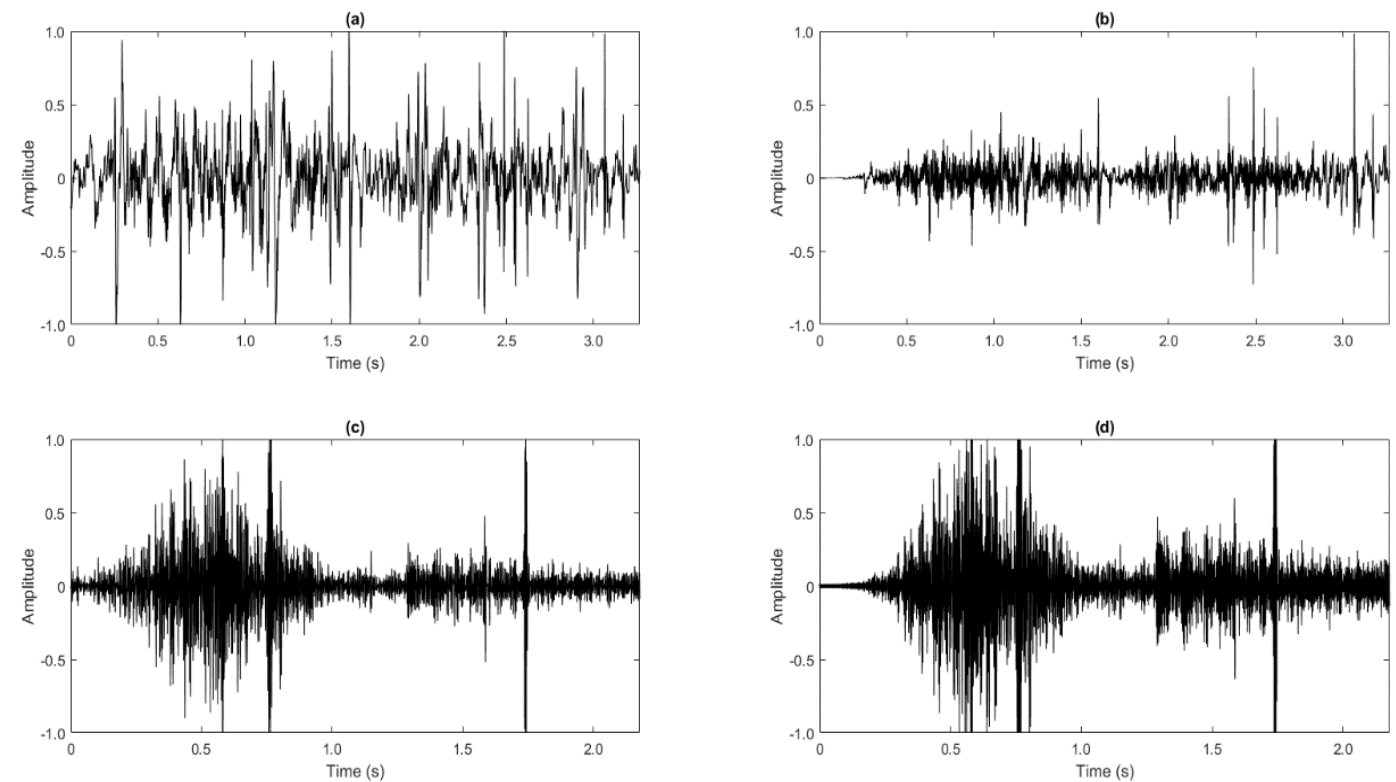

Figure 3. Respiratory sound cycle (inhale and exhale) for abnormal sound (a) before and (b) after filtering and for normal (c) before and (d) after filtering

\subsection{Signal decomposition using discrete wavelet transforms and feature extraction}

Wavelet transform provides time-frequency representation of a signal. Discrete wavelet transform can be written as [17],

$$
\psi_{j, k}(t)=2^{j / 2} \psi\left(2^{j} t-k\right), \quad j, k \in Z
$$


where $\psi$ is the wavelet function or the mother wavelet. $j$ is a positive value defines the scaling and $b$ is a real number that defines the shifting. Two mother wavelets namely Haar and $\mathrm{db} 7$ have been used for the signal decomposition. The decomposition of the signal using discrete wavelet transform involve convolution operation given as,

$$
\begin{aligned}
& y_{\text {high }}[k]=\sum_{n} x[n] \cdot h[2 k-n] \\
& y_{\text {low }}[k]=\sum_{n} x[n] \cdot g[2 k-n]
\end{aligned}
$$

where $h[$.$] is the discrete mother wavelet and in this case, it is the high pass filter and g[$ [.] for low pass filter. After the pre-processing stage, the signal was decomposed at seven levels using discrete wavelet transform to obtain prominent information at different frequency bands. The signal was passed through a high pass filter and a low pass filter followed by subsampling by 2 . The detail, $y_{\text {high }}\left(D_{1}, D_{2}, \ldots, D_{7}\right)$ and approximation, $y_{\text {low }}\left(A_{1}, A_{2}, \ldots, A_{7}\right)$ coefficients were obtained after the subsampling through high pass and low pass filters, respectively. The process is repeated for successive level until the desired level as shown in Figure 4. There are three frequency bands $\left(D_{4}, D_{5}, D_{6}\right)$ that have high amplitude of detail coefficients greater than 1 . These bands contain most information about the signal. Although the amplitude for $D_{3}$ and D7 was not so high compared to $D_{4}, D_{5}$ and $D_{6}$, they will be included in the features extraction as some information of the crackles may contain in these frequency bands. The frequency content for crackles is 100 to $2000 \mathrm{~Hz}$ or higher [5]. Therefore, five frequency bands $\left(D_{3}, D_{4}, D_{5}, D_{6}, D_{7}\right)$ were selected for feature extraction. Mean, standard deviation and maximum power spectral density (PSD) of detail coefficients of these five bands were calculated using MATLAB. These bands have frequency range from $86.13 \mathrm{~Hz}$ to $2756.25 \mathrm{~Hz}$.

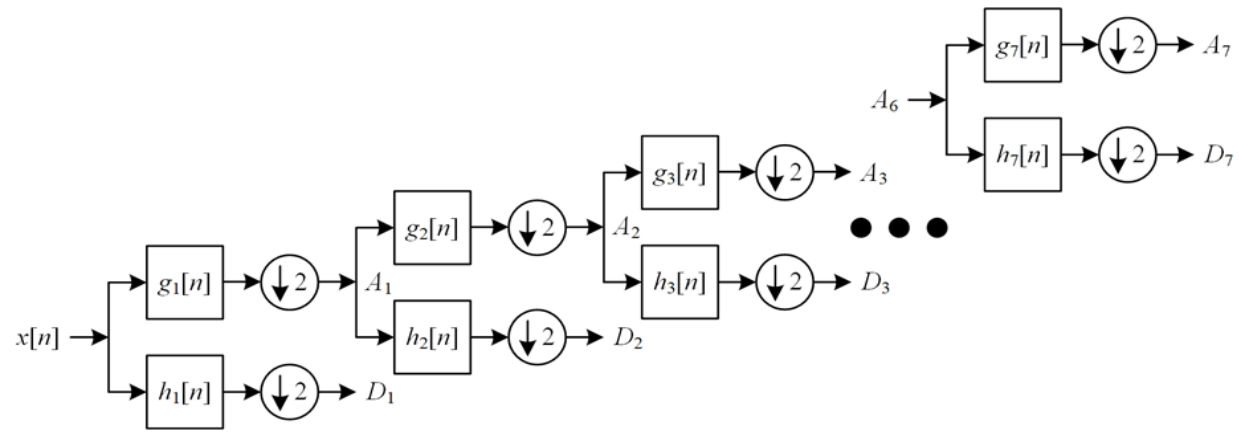

Figure 4. Signal decomposition process using DWT to obtain detail coefficients at seven level

\subsection{Classification using $\mathrm{ANN}$ and performance evaluation}

Neural networks consist of nodes which inspired by the neurons in the human brain of nervous system [18]. The network is built based on three layers namely input, hidden and output connected via nodes. The hidden layer can be more than one. Every node in the hidden layer is connected to all input (features) and on the other side of the node is connected to all output (class). Each connection between the input and the node carry a weightage. Aggregation of the input multiplied with the weightage is fed into activation function to obtain a new value as input to next layer. The most common activation function is sigmodal function. In this study, ANN has been employed as classifier using MATLAB to classify inputs into a set of target output that were initialized as matrix [1 0 ] for normal and [0 1] for crackles. In the training stage of the neural network, back propagation algorithm was used to adjust weights in the network if the predicted output does not match with the target output.

The Neural Network used in this study is multilayer feed forward neural network (MLFNN) trained with Backpropagation (BP). The optimal goal of backpropagation is to have minimal error that is relative to having outputs closer to the target. Assuming the data inputs are represented by $X i$ and weights by $W$; the detailed explanation is based on the Figure 5.

The neurons in each layer are fully connected to the neurons in the next layer, from layer $i$ to $j$ to $k$. Suppose that the network is designed with only one hidden layer neurons and generate only one output. Wij is the weight that connects the $i$ th neuron from input layer to the $j$ th neuron in the output layer, whereas $W j k$ is the weight that connects the $j$ th neuron from hidden layer to the $k$ th neuron in the output layer. In the BP algorithm, the generalization of delta rule involves two phases, which are the forward phase and the backward phase [19]. 


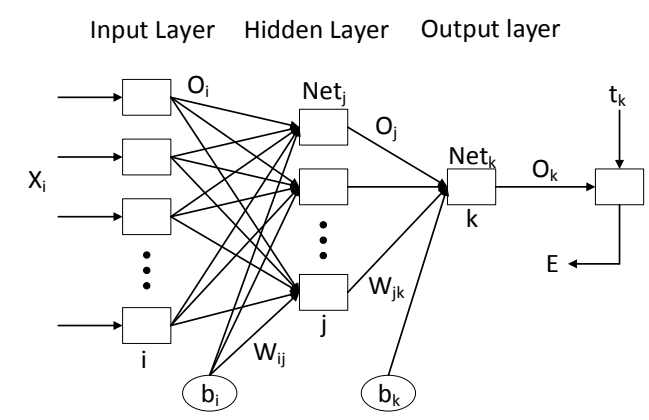

Figure 5. Signal Multilayer Perceptron with Backpropagation

The forward phase: For hidden layer output, consider

$$
O_{j}=\Phi\left(N e t_{j}\right)
$$

where

$$
N e t_{j}=\sum_{j} W_{i j} O_{i}+b_{j}
$$

where $b_{j}$ is the bias of the hidden node and can be set to zero, $\Phi$ is the sigmoid activation function. For output layer $k$, the network output is given as;

$$
O_{k}=\Phi\left(N e t_{k}\right)
$$

where

$$
\operatorname{Net}_{k}=\sum_{k} W_{j k} O_{k}+b_{k}
$$

The backward phase between output and hidden layer: The backward phase includes the calculation of the signal error and the weight update of the network. The network error ' $E$ ' is developed as follow:

$$
E=t_{k}-O_{k}
$$

where $t_{k}$ is the desired output and $O_{k}$ is the output of network which the output of the output layer. The objective is to find the set of parameters that minimize the sum of the squared of the error function, where the average sum squared error of the network is defined as;

$$
E=\frac{1}{N} \sum_{k=1}^{N}\left(t_{k}-O_{k}\right)^{2}=\frac{1}{N} \sum_{k=1}^{N} E^{2}
$$

where $\mathrm{N}$ is the total number of training pattern, $E$ is the error function to be minimized. The network weight update between the hidden layer $j$ and output layer $k$ is given by;

$$
W_{j k}^{\text {new }}=W_{j k}^{\text {old }}+\Delta W_{j k}
$$

where

$$
\Delta W_{j k}=\left.\eta \nabla E\right|_{W_{j k}}
$$

$\eta$ is the learning rate, $\left.\nabla E\right|_{W_{j k}}$ is the gradient of the cos function.

The backward phase between hidden layer and input layer: Adjusting between hidden layer and the input layer by:

$$
W_{i j}^{\text {new }}=W_{i j}^{\text {old }}+\Delta W_{i j}
$$


Therefore, the new weight update is;

$$
W_{i j}^{n e w}=W_{i j}^{o l d}+\eta \delta_{j} O_{i}
$$

\subsection{Performance evaluation}

The output of the algorithm was evaluated using the value of true positive (TP), true negative (TN), false positive (FP) and false negative (FN) to determine the sensitivity, specificity and accuracy using a suitable statistical analysis as shown in Equations (14), (15) and (16) [20].

$$
\begin{aligned}
& \text { Sensitivity }=\frac{T P}{T P+F N} \\
& \text { Specificity }=\frac{T N}{T N+F P} \\
& \text { Accuracy }=\frac{T P+T N}{T P+T N+F P+F N}
\end{aligned}
$$

\section{RESULTS AND DISCUSSION}

Most classification problems can be solved by only two hidden layers in ANN architecture [18], [22]. In this study, two hidden layers were employed in the architecture to classify between crackles and normal respiratory sounds. There were sixty crackles and sixty normal sounds used as samples. The samples are randomly divided into $70 \%$ for training, $15 \%$ for testing and $15 \%$ for validation. Eleven ANN models with a different number of nodes were chosen to be used in the training, validating and testing. For every chosen node, the data was retrained five times and the classification results for training, validation, and testing are tabulated in Table 1 . This is based on the best result obtained for testing.

As can be seen in Table 1 and Table 2, the best classification percentage was obtained when using 15 nodes and 10 nodes at the hidden layer for $\mathrm{db} 7$ and Haar, respectively. From the obtained results, db7 manage to get $100 \%$ correct classification for both test and validation stage which was very good as it achieved the perfect optimization. While for Haar, it only can obtain $100 \%$ correct classification at either validation or test stage. From the percentage obtained, test stage was the most important criteria that need to be a focus on because it helps in assessing the performance based on generalization and predictive power. The number of epoch for both $\mathrm{db} 7$ and Haar was not more than 20 which means it shows a good performance. The lower the number of epoch, the better the performance and the quicker it can achieve the best optimization.

For evaluation on classification performance, the percentage of sensitivity, specificity and accuracy was calculated based on Equations (14), (15) and (16) using the value of TP (correctly classified as crackles), TN (correctly classified as normal), FP (incorrectly classified as crackles) and FN (incorrectly classified as normal) as tabulated in Table 3 and Table 4 for $\mathrm{db} 7$ and Haar, respectively. From these tables, db7 based shows a better performance than Haar with $100 \%$ sensitivity, specificity and accuracy for testing and validation stages. As for Haar, only testing stage shows the perfect $100 \%$ for all sensitivity, specificity and accuracy. However, the percentage of accuracy for Haar was still good for classification of crackles and normal sounds.

Table 1. Performance of Various ANN Model for $\mathrm{db} 7$

\begin{tabular}{ccccc}
\hline $\begin{array}{c}\text { ANN model } \\
\text { (Input-Nodes-Output) }\end{array}$ & $\begin{array}{c}\text { No. epoch } \\
\text { (best validation) }\end{array}$ & $\begin{array}{c}\text { Training } \\
(\%)\end{array}$ & $\begin{array}{c}\text { Validation } \\
(\%)\end{array}$ & $\begin{array}{c}\text { Testing } \\
(\%)\end{array}$ \\
\hline $15-10-2$ & 14 & 90.5 & 94.4 & 100 \\
$15-11-2$ & 12 & 92.9 & 88.9 & 94.4 \\
$15-12-2$ & 9 & 89.3 & 83.3 & 94.4 \\
$15-13-2$ & 18 & 91.7 & 94.4 & 94.4 \\
$15-14-2$ & 14 & 91.7 & 94.4 & 100.0 \\
$15-15-2$ & 5 & 90.5 & 100.0 & 100.0 \\
$15-35-2$ & 2 & 90.5 & 83.3 & 94.4 \\
$15-55-2$ & 8 & 91.7 & 88.9 & 100.0 \\
$15-75-2$ & 3 & 91.7 & 94.4 & 88.9 \\
$15-95-2$ & 8 & 90.5 & 94.4 & 100 \\
$15-115-2$ & 13 & 94.0 & 88.9 & 94.4 \\
\hline
\end{tabular}


Table 2. Performance of Various ANN Model for Haar

\begin{tabular}{lcccc}
\hline $\begin{array}{c}\text { ANN model } \\
\text { (Input-Nodes-Output) }\end{array}$ & $\begin{array}{c}\text { No. epoch } \\
\text { (best validation) }\end{array}$ & $\begin{array}{c}\text { Training } \\
(\%)\end{array}$ & $\begin{array}{c}\text { Validation } \\
(\%)\end{array}$ & $\begin{array}{c}\text { Testing } \\
(\%)\end{array}$ \\
\hline $15-10-2$ & 7 & 89.3 & 94.4 & 100.0 \\
$15-11-2$ & 4 & 90.5 & 83.3 & 100.0 \\
$15-12-2$ & 9 & 89.3 & 88.9 & 100.0 \\
$15-13-2$ & 6 & 90.5 & 94.4 & 94.4 \\
$15-14-2$ & 13 & 89.3 & 94.4 & 94.4 \\
$15-15-2$ & 5 & 90.5 & 83.3 & 94.4 \\
$15-35-2$ & 4 & 89.3 & 94.4 & 94.4 \\
$15-55-2$ & 6 & 90.5 & 88.9 & 100.0 \\
$15-75-2$ & 12 & 86.9 & 88.9 & 100.0 \\
$15-95-2$ & 8 & 90.5 & 100.0 & 88.9 \\
$15-115-2$ & 7 & 91.7 & 88.9 & 94.4 \\
\hline
\end{tabular}

Table 3. Evaluation Performance of ANN Classification of Crackles and Normal Sounds using db7

\begin{tabular}{lccccccc}
\hline \multicolumn{1}{c}{ Stage } & TP & TN & FP & FN & Sensitivity (\%) & Specificity (\%) & Accuracy (\%) \\
\hline Training & 38 & 38 & 4 & 4 & 90.5 & 90.5 & 90.5 \\
Test & 9 & 9 & 0 & 0 & 100.0 & 100.0 & 100.0 \\
Validation & 9 & 9 & 0 & 0 & 100.0 & 100.0 & 100.0 \\
All & 56 & 56 & 4 & 4 & 93.3 & 93.3 & 93.3 \\
\hline
\end{tabular}

Table 4. Evaluation Performance of ANN Classification of Crackles and Normal Sounds using Haar

\begin{tabular}{lccccccc}
\hline \multicolumn{1}{c}{ Stage } & TP & TN & FP & FN & Sensitivity (\%) & Specificity (\%) & Accuracy (\%) \\
\hline Training & 39 & 36 & 4 & 5 & 88.6 & 90.0 & 89.3 \\
Test & 11 & 7 & 0 & 0 & 100.0 & 100.0 & 100.0 \\
Validation & 6 & 11 & 0 & 1 & 85.7 & 100.0 & 94.4 \\
All & 56 & 54 & 4 & 6 & 90.3 & 96.6 & 91.7 \\
\hline
\end{tabular}

\section{CONCLUSION}

These preliminary results towards the development of screening method for lung cancer using computerized have resulted with positive outcomes. Nevertheless, other factors such as age, smoking habit, ambient air pollution and occupational exposure need to be considered when interpreting the results in future. These factors can be added as features to the classifier. In this study, normal and crackles respiratory sounds have successfully been classified using ANN with backpropagation consists of two hidden layers. Both mother wavelets, Haar, and db7 can provide distinctive pattern needed as features in learning algorithm with some statistical and signal strength formulation such as mean, standard deviation, and PSD.

\section{ACKNOWLEDGEMENTS}

The authors would like to acknowledge the Ministry of Higher Education Malaysia (MOHE) for funding this research project through Fundamentals Research Grant Scheme (FRGS) [Ref.:FRGS16-0670566]. We also would like to thank Dr. Rozita Abdul Malik and Dr. Adlinda Alip of UMMC for their consultation and advice on lung cancer related issues

\section{REFERENCES}

[1] Steward BW, Wild CP. World cancer report 2014. Health. 2017 Oct 24.

[2] Azizah AM, Nor Saleha IT, Noor Hashimah A, Asmah ZA, Mastulu W, "Malaysian national cancer registry report 2007-2011", Ministry of Health Malaysia, 2016.

[3] Hoffman RM, Sanchez R, "Lung Cancer Screening”, Medical Clinics, 2017 Jul 1, vol. 101, no. 4, pp. 769-85.

[4] Andolfi M, Potenza R, Capozzi R, Liparulo V, Puma F, Yasufuku K, "The role of bronchoscopy in the diagnosis of early lung cancer: a review", Journal of thoracic disease, 2016 Nov, vol. 8, no. 11, 3329.

[5] Gurung A, Scrafford CG, Tielsch JM, Levine OS, Checkley W, "Computerized lung sound analysis as diagnostic aid for the detection of abnormal lung sounds: A systematic review and meta-analysis", Respiratory medicine, 2011 Sep 1, vol. 105, no. 9, pp. 1396-403.

[6] Gnitecki J, Moussavi ZM, "Separating heart sounds from lung sounds", IEEE Engineering in medicine and biology magazine, 2007 Jan 1, vol. 26, no.1, 20. 
[7] Hossain I, Moussavi Z, "An overview of heart-noise reduction of lung sound using wavelet transform based filter", In Engineering in Medicine and Biology Society, 2003, Proceedings of the 25th Annual International Conference of the IEEE 2003 Sep 17 (vol. 1, pp. 458-461), IEEE.

[8] Lakhe A, Sodhi I, Warrier J, Sinha V, "Development of digital stethoscope for telemedicine", Journal of medical engineering \& technology, 2016 Jan 2, vol. 40, no. 1, pp. 20-24.

[9] Xie S, Jin F, Krishnan S, Sattar F, "Signal feature extraction by multi-scale PCA and its application to respiratory sound classification", Medical \& biological engineering \& computing, 2012 Jul 1, vol. 50, no. 7, pp. 759-768.

[10] Jin F, Sattar F, Goh DY, "New approaches for spectro-temporal feature extraction with applications to respiratory sound classification", Neurocomputing, 2014 Jan 10, 123, pp. 362-371.

[11] Gögüş FZ, Karlık B, Harman G, "Classification of asthmatic breath sounds by using wavelet transforms and neural networks", International Journal of Signal Processing Systems, 2015 Dec, vol. 3, no. 2, pp. 106-111.

[12] Pasterkamp H, Kraman SS, Wodicka GR, "Respiratory sounds: advances beyond the stethoscope", American journal of respiratory and critical care medicine, 1997 Sep 1, vol. 156, no. 3, pp. 974-987.

[13] İçer S, Gengeç Ş, "Classification and analysis of non-stationary characteristics of crackle and rhonchus lung adventitious sounds", Digital Signal Processing, 2014 May 1, vol. 28, no. 18-27.

[14] Rizal A, Hidayat R, Nugroho HA, "Pulmonary crackle feature extraction using tsallis entropy for automatic lung sound classification", In Biomedical Engineering (IBIOMED), International Conference on 2016 Oct 5 (pp. 1-4), IEEE.

[15] Chen CH, Huang WT, Tan TH, Chang CC, Chang YJ, "Using k-nearest neighbor classification to diagnose abnormal lung sounds", Sensors, 2015 Jun 4, vol. 15, no. 6, pp. 13132-13158.

[16] Li J, Hong Y, "Crackles detection method based on time-frequency features analysis and SVM", In Signal Processing (ICSP), 2016 IEEE 13th International Conference on 2016 Nov 6 (pp. 1412-1416), IEEE.

[17] Cohen A, Kovacevic J. Wavelets, "The mathematical background", Proceedings of the IEEE, 1996 Apr, vol. 84, no. 4 , pp. 514-522.

[18] Lapedes AS, Farber RM, "How neural nets work", In Neural information processing systems 1988 (pp. 442-456).

[19] R. F. Olanrewaju, O. Khalifa, and K. N. A. Latif, "Computational Intelligence: It's Application in Digital Watermarking”, Middle-East J. Sci. Res., vol. 13, pp. 25-30, 2013.

[20] Baratloo, Alireza, Mostafa Hosseini, Ahmed Negida, and Gehad El Ashal, "Part 1: simple definition and calculation of accuracy, sensitivity and specificity", 2015, pp. 48-49.

[21] Gunawan, T.S., and Kartiwi, M., "n the Comparison of Line Spectral Frequencies and Mel-Frequency Cepstral Coefficients Using Feedforward Neural Network for Language Identification", Indonesian Journal of Electrical Engineering and Computer Science, vol. 10, no. 1, pp. 168-175, April 2018.

\section{BIOGRAPHIES OF AUTHORS}
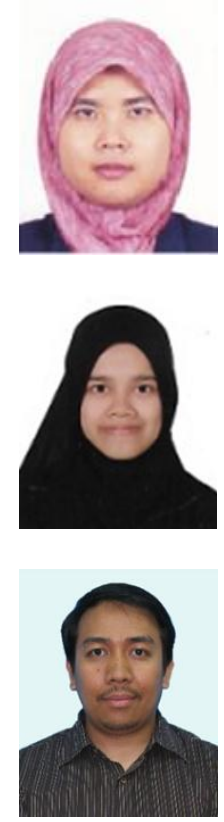

Noreha Abdul Malik received her BEng in Medical Electronics from University of Technology Malaysia (2001) and later pursued her MEng in Communication and Computer Engineering at National University of Malaysia (2004). She later received her PhD in Electronics and Electrical Engineering from University of Southampton, United Kingdom (2011). She is currently an assistant professor at International Islamic University Malaysia (IIUM). Her research interests are in biomedical signal processing and biomedical applications. She is a member of Institute of Engineers Malaysia (IEM) and Board of Engineer Malaysia (BEM).

Wardati Idris received her BEng degree in Communication Engineering in 2018 from the International Islamic University Malaysia. Her research interests include signal processing in biomedical field.

Teddy Surya Gunawan received his BEng degree in Electrical Engineering with cum laude award from Institut Teknologi Bandung (ITB), Indonesia in 1998. He obtained his M.Eng degree in 2001 from the School of Computer Engineering at Nanyang Technological University, Singapore, and $\mathrm{PhD}$ degree in 2007 from the School of Electrical Engineering and Telecommunications, The University of New South Wales, Australia. His research interests are in speech and audio processing, biomedical signal processing and instrumentation, image and video processing, and parallel computing. He is currently an IEEE Senior Member (since 2012), was chairman of IEEE Instrumentation and Measurement Society - Malaysia Section (2013 and 2014), Associate Professor (since 2012), Head of Department (2015-2016) at Department of Electrical and Computer Engineering, and Head of Programme Accreditation and Quality Assurance for Faculty of Engineering (since 2017), International Islamic University Malaysia. He is Chartered Engineer (IET, UK) and Insinyur Profesional Madya (PII, Indonesia) since 2016. 


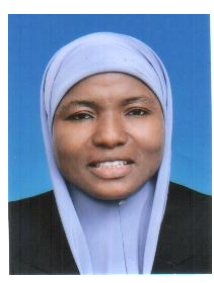

Rashidah Funke Olanrewaju received her BSc. Hons. Computer Science from University Putra Malaysia, majoring in Software Engineering in 2002, M.Sc. in Computer and Information Engineering, majoring in Information Engineering from International Islamic University Malaysia (IIUM) in 2006. She later received her PhD (Engineering) in August 2011 with specialization in Information Security in Digital Image Processing. She also received a Postgraduate Diploma in Islamic Studies (DIS) from IIUM in 2001. She is currently an Assistant professor at International Islamic University Malaysia (IIUM). Her research interests are Information Security, Application of Artificial Intelligence in Bioenvironmental Systems, Computer Architecture and Design, Telemedicine Systems, Image Processing and Cloud Computing. She is a Senior member of Institute of Electrical and Electronics Engineer (SMIEEE), Computer Society, Women in Engineering (WIE), Member (IET, UK)Arab Research Institute in Science and Engineering (ARISE), Nigeria Computer Society (NCS) Malaysian Society for Cryptology Research (MSCR), Cloud Computing, Virtualization and Disaster Recovery in Nigeria Network.

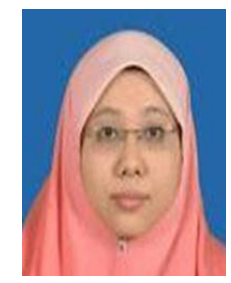

S. Noorjannah Ibrahim has a PhD. in Electrical and Computer Engineering from the University of Canterbury, New Zealand. She specializes in micro-nano fabrication technology particularly in pattern transfer technique, metal deposition, microfluidic design, BIOMEMS, MEMS and biomedical application. Currently, her research interest is in the area of respiratory (biomedical) sensor and IoT applications. She has been an academic since 2001 and has considerable teaching experience in undergraduate level and postgraduate, ranging from the fundamentals course (electronics) to the more specialist topics such as wireless technology and MEMS. To date, she works as an Assistant Professor at the Department of Electrical \& Computer Engineering, Kulliyyah Of Engineering, International Islamic University Malaysia (IIUM). She is a senior member of IEEE, Secretary of IEEE Electron Devices Society (EDS) Malaysia Chapter and a member of Institute of Engineers Malaysia (IEM). 